\title{
Decoy receptor-3 regulates inflammation and apoptosis via PI3K/AKT signaling pathway in coronary heart disease
}

\author{
XINJING CHEN $^{1,2}$, REHUA WANG ${ }^{2}$, WEI CHEN ${ }^{2}$, LI LAI $^{3}$ and ZHILIANG LI ${ }^{1}$ \\ ${ }^{1}$ Department of Cardiology, Zhujiang Hospital, Southern Medical University, Guangzhou, Guangdong 510280; \\ ${ }^{2}$ Department of Cardiology; ${ }^{3}$ Fujian Key Laboratory of Cardiovascular Disease, Fujian Provincial Hospital, \\ Fujian Medical University, Fuzhou, Fujian 350001, P.R. China
}

Received November 4, 2017; Accepted November 1, 2018

DOI: $10.3892 /$ etm.2019.7222

\begin{abstract}
Coronary heart disease is a disease characterized by coronary artery atherosclerosis lesions caused by vascular cavity stenosis, occlusion, myocardial ischemia, hypoxia or necrosis. Previous studies have demonstrated that decoy receptor-3 (DCR-3) can act as a pleiotropic immunomodulation for enhancing angiogenesis, which may be associated with the progression of coronary heart disease. In the present study, ELISA assay was used to investigate the plasma concentration level of DCR-3 in patients with coronary heart disease. The mRNA and protein level of DCR-3 in myocardial cells were determined by reverse transcription-quantitative polymerase chain reaction and western blotting, respectively. The role and molecular mechanism of DCR-3 was also evaluated in myocardial cells in mice with coronary heart disease. The role of small interfering RNA that targeted phosphoinositide 3-kinase (PI3K) in DCR-3 mediated apoptosis was confirmed by terminal deoxynucleotidyl-transferase-mediated dUTP nick end labeling and immunofluorescence. C57BL/6 mice with coronary heart disease were used to evaluate the efficacy of DCR-3 on inflammation and apoptosis. The data indicated that plasma concentration level of DCR-3 was downregulated in mice with coronary heart disease and that DCR-3 administration improved symptoms of coronary heart disease and prolonged survival of mice with coronary heart disease. In addition, it was demonstrated that DCR-3 treatment suppressed the inflammatory response and apoptosis of myocardial cells. Circulating DCR-3 concentration levels may be identified as a predictor of coronary heart disease and prognosis of coronary heart disease. Notably, it was also demonstrated that DCR-3 inhibited inflammatory factor expression levels by regulation of the PI3K/protein kinase B (AKT) signaling pathway.
\end{abstract}

Correspondence to: Professor Zhiliang Li, Department of Cardiology, Zhujiang Hospital, Southern Medical University, 253 Industrial Avenue, Guangzhou, Guangdong 510280, P.R. China E-mail: zhiliangli@yeah.net

Key words: coronary heart disease, decoyreceptor-3, inflammation, apoptosis
Taken together, these results indicate that increasing circulating DCR-3 plasma concentration is associated with degree of coronary heart disease, suggesting that DCR-3 may be a promising drug for the treatment of coronary heart disease via regulating inflammation and apoptosis through the PI3K/AKT signaling pathway.

\section{Introduction}

Coronary heart disease is a generic term for coronary arteries atherosclerosis lesions caused by a large number of factors, such as vascular cavity stenosis, occlusion, myocardial ischemia, hypoxia, necrosis, inflammation and apoptosis of myocardial cells $(1,2)$. It has been demonstrated that reduced blood pressure and cholesterol are important factors in reducing deaths from coronary heart disease (3). In addition, a large number of proteins are associated with the protective effects for host against myocardial ischemia and reperfusion injury through modulating myocardial apoptosis and inflammation $(4,5)$. Furthermore, inflammation and apoptosis contribute to the initiation and development of coronary heart disease, which have been considered as the prognostic indicators for patients with coronary heart disease following drug treatment in clinical settings $(6,7)$. Therefore, a number of potential strategies to relieve or mitigate the apoptosis of myocardial cells have been proposed to protect the heart against coronary heart disease, such as ameliorating the apoptosis of cardiac cells mediated by endothelial stem cell (8), or activating the signaling pathway to promote the expression of anti-apoptosis proteins and decrease pro-apoptosis proteins to protect the heart (9).

Coronary heart disease is the leading cause of mortality worldwide and is closely associated with metabolism disorders of endogenous substances (10). A growing number of studies have reported that apoptosis of myocardial cells has a crucial role in the progression of cardiovascular diseases (11-13). Apoptosis of leukocytes has been considered as a marker of neutrophil-endotheliocyte interaction in coronary heart disease (14). Expression of apoptosis factors has become an important reference of surgical results following coronary stent implantation in coronary heart disease patients determined by characteristics of neutrophil cluster of differentiation (CD)11b, platelet CD62P, endothelin, monocyte CD11b, and neutrophil 
CD178 in patients with coronary heart disease prior to and following primary coronary stenting (15).

Inflammation as the cause of coronary heart disease has been reviewed previously (16). Expression levels of inflammation cytokines including interleukin (IL)-6, tumor necrosis factor (TNF)- $\alpha$ and IL-1 in patients with coronary heart disease may also be associated with the progression pathogenesis (17). These reports indicate that inhibition of inflammation contributes to the treatment of coronary heart disease. In the present study, the inhibitory effects of decoy receptor-3 (DCR-3) on inflammation were investigated.

It has been demonstrated that DCR-3 can act as a pleiotropic immunomodulator for enhancing angiogenesis, which may be associated with the progression of coronary heart disease (18). DCR-3 is well known as soluble receptor TR6, which belongs to the TNFR superfamily and is constituted by a 300-aa polypeptide $(19,20)$. DCR-3 has been identified as the most common biomarker of tumor deterioration in cancer patients by a previous meta-analysis (21). However, no previous report has focused on the clinical significance of DCR-3 expression levels in coronary and peripheral blood of coronary heart disease.

In the present study, the DCR-3 concentration levels were investigated in patients with coronary heart disease. The efficacy and molecular mechanism of DCR-3 were evaluated in mice with coronary heart disease. Inflammation and apoptosis of myocardial cells were investigated in mice with coronary heart disease following treatment with DCR-3. The phosphoinositide 3-kinase (PI3K)/protein kinase B (AKT) signaling pathway was also analyzed in DCR-3-treated myocardial cells.

\section{Materials and methods}

Ethics statement. A total of 242 patients (age, 27-56 years; female:male, 1:1.1) with coronary heart disease and 103 healthy volunteers age, (28-63 years; female:male, 1:1.1) were recruited from Department of Cardiology, Zhujiang Hospital, Southern Medical University (Guangzhou, China) between January and May 2016 for analyzing serum levels of DCR-3. The patients were classified into four groups. Health group contained participants without angina. Primary group participants who only suffered angina during intense, rapid or prolonged physical activity or exercise. Moderate group were slightly limited in daily activities, as angina occurred following brisk walking, going upstairs, walking in cold air or after mood swings. Severe group were under great limitation and suffered angina after even mild exercise. The present study was approved by the Ethics Committee of Southern Medical University (Guangzhou, China). All patients provided written informed consent prior to experiments. Primary, moderate and severe coronary heart disease was diagnosed using 2014 ACC/AHA Recommendations for the management of NSTE ACS (22).

Cell culture. Cardiac fibroblasts were harvested from C57BL/6J mice as described previously (23). A total of 50 female C57BL/6J (age, 8 weeks; weight, 28-32 g) mice were purchased from OrientBio, Inc. (Seongnam, Korea). All mice were given free access to food and water, and housed at $23^{\circ} \mathrm{C}$ with $50 \%$ humidity and a 12 -h artificial light/dark cycle. Briefly, mice were sacrificed under $20 \mathrm{mg} / \mathrm{kg}$ subcutaneous meperidine anesthesia (24). Harvested hearts were washed with PBS and heart tissues were digested with digestion buffer prepared with $0.05 \%(\mathrm{w} / \mathrm{v})$ collagenase type II (Worthington Biochemical Corporation, Lakewood, NJ, USA) and $0.06 \%$ (w/v) pancreatin (Sigma-Aldrich; Merck KGaA, Darmstadt, Germany) in PBS for $10 \mathrm{~min}$ at $37^{\circ} \mathrm{C}$. The supernatant was collected and filtrated with a $70-\mu \mathrm{m}$ filter and collected cells were centrifuged at $100 \mathrm{x} \mathrm{g}$ for $4 \mathrm{~min}$ at $25^{\circ} \mathrm{C}$ and resuspended in culture medium: RPMI-1640 medium (Sigma-Aldrich; Merck KGaA) with penicillin-streptomycin (Thermo Fisher Scientific, Inc., Waltham, MA, USA) at $37^{\circ} \mathrm{C}$ for $12 \mathrm{~h}$. Following washing out digestion buffer with RPMI-1640 medium twice, cells were seeded on cell culture dishes and incubated for $1 \mathrm{~h}$ at $37^{\circ} \mathrm{C}$. Following incubation, unattached cells were removed and attached cells were cultured with fresh culture medium supplemented with $10 \%$ fetal bovine serum (Sigma-Aldrich; Merck $\mathrm{KGaA}$ ). Cells were cultured in a $5 \% \mathrm{CO}_{2}$ incubator with a humidified atmosphere at $37^{\circ} \mathrm{C}$.

RNA isolation and reverse transcription-quantitative polymerase chain reaction $(R T-q P C R)$. Total RNA was extracted from cardiac fibroblasts using TRIzol reagent (Life Technologies; Thermo Fisher Scientific, Inc.). Quality of extracted mRNA was identified by Spectroscopy Elemental Isotope Analysis using NanoDrop Lite (Thermo Fisher Scientific, Inc.). According to the manufacturer's descriptions, total RNA $(1 \mu \mathrm{g})$ was reverse-transcribed to cDNA using the Transcriptor First Strand cDNA Synthesis kit (Invitrogen; Thermo Fisher Scientific, Inc.). The qPCR reaction was performed with a SYBR-Green detection system (Bio SYBR Green Master Mix; Takara Biotechnology Co., Ltd., Dalian, China). The following primers were synthesized by Invitrogen; Thermo Fisher Scientific, Inc.: DCR-3, forward 5'-CTCTTC CTCCCATGACAC-3' and reverse 5'-CTGGAAAGCCAC AAAGTC-3'; and $\beta$-actin, forward 5'-CGTGAAAAGATG ACCCAGATCA-3' and reverse 5'-CAGCCTGGATGGCTA CGTACA-3'. PCR cycling conditions were performed at $95^{\circ} \mathrm{C}$ for $30 \mathrm{sec}$ and 45 cycles of $95^{\circ} \mathrm{C}$ for $5 \mathrm{sec}, 56.5^{\circ} \mathrm{C}$ for $10 \mathrm{sec}$ and $72^{\circ} \mathrm{C}$ for $10 \mathrm{sec}$. Relative mRNA expression changes were calculated by the $2^{-\Delta \Delta \mathrm{Cq}}$ method (25). The results are expressed as a fold-change compared with the $\beta$-actin control.

Small interfering RNA (siRNA) transfection. siRNA to target PI3K (Si-PI3K) and scrambled siRNA (Si-vector) were designed and synthesized by Shanghai GenePharma Co., Ltd. (Shanghai, China). The sequences were as follows: Si-PI3K, forward 5'-CCAACAACAGCAUGAACAAdTdT-3' and reverse 5'-UUGUUCAUGCUGUUGUUGGdTdT-3'; and Si-vector, forward 5'-AUGAACGUGAAUUGCUCA AdT dT-3' and reverse 5'-UUGAGCAAUUCACGUUCAUdTdT-3'. Cardiac fibroblasts isolated from experimental mice were seeded in 24-well plates and were cultured to $80 \%$ confluence. Then $1.25 \mu \mathrm{l} 25 \mu \mathrm{M}$ Si-PI3K or Si-vector were transfected to cells using Lipofectamine ${ }^{\mathrm{TM}}$ RNAi MAX (Invitrogen; Thermo Fisher Scientific, Inc.) according to the manufacturer's instructions. Si-PI3K and Si-vector were from Shanghai GenePharma Co., Ltd. (Shanghai, China). The interval between transfection and subsequent experiments was $24 \mathrm{~h}$. 
Syntax score. Syntax score of patients with coronary heart disease were calculated according to a previous study (26). The DCR-3 expression and syntax score were analyzed in patients with coronary heart disease with healthy volunteers as controls.

ELISA. In the protein detection assay, serum levels of DCR-3 were measured using an ELISA kit (DY142; Bio-Rad Laboratories, Inc., Hercules, CA, USA). The operating steps were conducted according to the manufacturer's instructions. The final results were recorded at $450 \mathrm{~nm}$ on an ELISA plate reader.

Western blotting. Myocardial cells harvested from mice were lysed in radioimmunoprecipitation assay buffer containing a phosphatase inhibitor and the protease inhibitor cocktail (Pierce; Thermo Fisher Scientific, Inc.). Protein concentrations were determined via bicinchoninic protein assay (Pierce; Thermo Fisher Scientific, Inc.). Equal amounts of proteins (40 $\mu \mathrm{g} /$ lane) were loaded and separated by $12 \%$ SDS-PAGE. The proteins were transferred to polyvinylidene difluoride membranes (EMD Millipore, Billerica, MA, USA). The membranes were then blocked for $1 \mathrm{~h}$ at $37^{\circ} \mathrm{C}$ with $10 \%$ bovine serum albumin (cat. no. 10735108001; Roche Applied Science, Penzburg, Germany). The following rabbit anti-mouse antibodies were used to incubate protein for $12 \mathrm{~h}$ at $4^{\circ} \mathrm{C}$ : DCR-3 (1:1,000, cat. no. ab8405), cardiac troponin (cTn)T (1:1,000; cat. no. ab45932), cTn1 (1:1,000; cat. no. ab210798), IL-6 (1:1,000; cat. no. ab83053), C-reactive protein 1 (CRP-1) (1:1,000; cat. no. ab211631), albumin (1:1,000; cat. no. ab207327), intercellular adhesion molecule (ICAM)-1 (1:1,000; cat. no. ab119817), vascular cell adhesion molecule (VCAM)-1 (1:1,000; cat. no. ab134047), PI3K (1:1,000; cat. no. ab40776), AKT (1:1,000; cat. no. ab8805), phosphorylated (p)AKT (1:1,000; cat. no. ab81283) and $\beta$-actin (1:2,000; cat. no. ab8226; all Abcam, Cambridge, UK). Horseradish peroxidase (HRP)-conjugated goat anti-rabbit antibody (1:5,000; cat. no. HAF019; Bio-Rad Laboratories, Inc.) was used as a secondary antibody for $2 \mathrm{~h}$ at $37^{\circ} \mathrm{C}$ and protein was detected using a western blotting Luminol reagent (cat. no. 12015218001; Sigma-Aldrich; Merck KGaA) for enhanced chemiluminescence. Lanes were observed using light microscopy (magnification, $\mathrm{x} 4$ ). The density of the bands was analyzed using Quantity One software, version 4.62 (Bio-Rad Laboratories, Inc.).

Immunofluorescence. Myocardial cells were fixed with formaldehyde solution (10\%) for $2 \mathrm{~h}$ at $37^{\circ} \mathrm{C}$ and processed according to standard procedures. The sections are blocked with $5 \%$ bovine serum albumin (Sigma-Aldrich; Merck KGaA) at $37^{\circ} \mathrm{C}$ for $15 \mathrm{~min}$. Cells were incubated with primary antibody against DCR-3 (1:1,000, cat. no. ab8405) for $12 \mathrm{~h}$ at $4^{\circ} \mathrm{C}$. Cells were then incubated with HRP-conjugated anti-rabbit antibodies (1:200; cat. no. 71623, Bio-Rad Laboratories, Inc.) for $2 \mathrm{~h}$ at $37^{\circ} \mathrm{C}$. Cells were washed and mounted with VectaShield mounting media with DAPI (Vector Laboratories, Inc., Burlingame, CA, USA) and kept in the dark at $4^{\circ} \mathrm{C}$ prior to microscopic analysis. All images (magnification, $\mathrm{x} 10$ ) were captured with a confocal microscope (Fluoview1000; Olympus Corporation, Tokyo, Japan).
Animal experiments. A total of 50 female C57BL/6J (age, 8 weeks; weight, 28-32 g) mice were purchased from OrientBio, Inc. (Seongnam, Korea). All mice were given free access to food and water, and housed at $23^{\circ} \mathrm{C}$ with $50 \%$ humidity with a 12-h artificial light/dark cycle. A total of 45 mice were fed with a high-fat diet to establish coronary heart disease model according to a previous study (27); 5 healthy mice and 5 model mice were used to demonstrated the changes in DCR-3 level due to coronary heart disease. The remaining 40 model mice were divided into two groups ( $n=20 /$ group) and received an intravenous injection of DCR-3 (10 mg/kg; Sigma-Aldrich; Merck KGaA) or PBS (Control). The treatments were repeated once daily for 10 days. Following 3 weeks, animals were sacrificed by cervical dislocation under anesthesia with $3 \%$ isoflurane (Sigma-Aldrich; Merck KGaA). The present study has been approved by the Committee for Experimental Animal Studies of Southern Medical University.

Histological assay. Cardiac slices isolated from mice with cardiac fibrosis were prepared and fixed in $4 \%$ paraformaldehyde for $30 \mathrm{~min}$ at $37^{\circ} \mathrm{C}$. Cardiac slices were performed using an avidin-biotin-peroxidase technique. Paraffin-embedded tissue sections were prepared, heated at $65^{\circ} \mathrm{C}$ to melt the paraffin and dewaxed to water with xylene, ethyl alcohol (100, 95,85 and $75 \%$ ) and water successively. Epitope retrieval was performed for further analysis. The paraffin sections $(4 \mu \mathrm{m})$ were incubated with hydrogen peroxide $(3 \%)$ for $10 \mathrm{~min}$, and were blocked with $5 \%$ bovine serum albumin (Sigma-Aldrich; Merck $\mathrm{KGaA}$ ) for $10 \mathrm{~min}$ at $37^{\circ} \mathrm{C}$. Finally, the sections were incubated with rabbit anti-mouse anti-DCR-3 (cat. no. ab8405; Abcam) diluted with PBS (1:1,000) or anti-major histocompatibility complex I antibodies (cat. no. ab185706; Abcam) diluted with PBS $(1: 1,000)$, for $12 \mathrm{~h}$ at $4^{\circ} \mathrm{C}$ following blocking with $5 \%$ bovine serum albumin (Sigma-Aldrich; Merck KGaA). Following rinsing, sections were incubated in the presence of a biotinylated horse anti-rabbit antibody (cat. no. a0545; 1:500; Chemicon; Merck KGaA) for $2 \mathrm{~h}$ at $37^{\circ} \mathrm{C}$. Sections were washed and observed (magnification, x10) using fluorescent video microscopy (BZ-9000; Keyence Corporation, Osaka, Japan).

Terminal deoxynucleotidyl-transferase-mediated dUTP nick end labeling (TUNEL) assay. The TUNEL assay was used for the analysis of apoptosis of myocardial tissue in experimental mice at $37^{\circ} \mathrm{C}$ following simvastatin treatment (10 mg/kg/day; Sigma-Aldrich; Merck KGaA) or an equivalent dose of PBS. Procedures were performed as previously described (27). Briefly, 4- $\mu \mathrm{m}$ tissue sections were fixed with $4 \%$ paraformaldehyde at $37^{\circ} \mathrm{C}$ followed by permeabilization with $0.1 \%$ Triton $\mathrm{X}-100$ for $1 \mathrm{~h}$ at $37^{\circ} \mathrm{C}$. Subsequently, tissue sections or myocardial cells $\left(1 \times 10^{6}\right)$ were stained with TUNEL reaction mixture (Sigma-Aldrich; Merck KGaA) at $37^{\circ} \mathrm{C}$ for $2 \mathrm{~h}$. Tissue sections and myocardial cells were washed 3 times in TBS-Tween-20. TUNEL assays were conducted using a TUNEL fluorescence FITC kit (Roche Diagnostics, Indianapolis, IN, USA) according to the manufacturer's instructions. Samples were mounted with neutral gum. Images in 6 fields of view (magnification, $x 400$ ) were captured using a Zeiss LSM 510 confocal microscope (Zeiss AG, Oberkochen, Germany) at a wavelength of $488 \mathrm{~nm}$. 

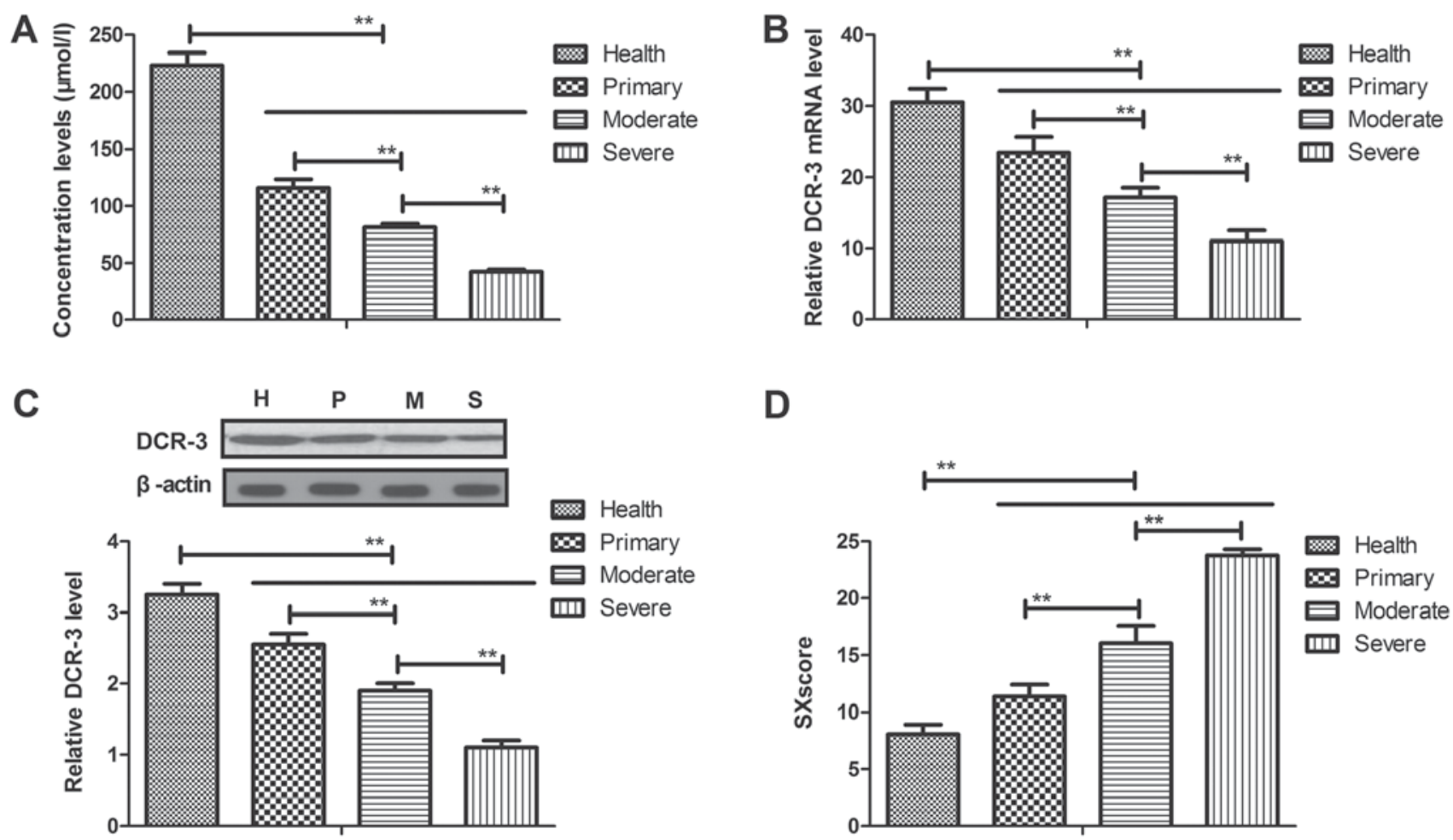

Figure 1. Analysis of DCR-3 expression levels in patients with coronary heart disease. (A) Plasma concentration levels of DCR-3 in patients with coronary heart disease. (B and C) DCR-3 mRNA and protein expression in myocardial cells were analyzed in patients with coronary heart disease. H, health; P, primary; M, moderate; S, severe. (D) Association between SXscore and the severity of coronary heart disease. Data are presented as the mean \pm standard deviation of three independent experiments. ${ }^{* *} \mathrm{P}<0.01$. DCR-3, decoy receptor-3; SXscore, syntax score.

Statistical analysis. Results are expressed as the mean + standard deviation. All data were analyzed using SPSS software (version 19.0; IBM Corp., Armonk, NY, USA). Comparisons between two groups and among multiple groups were conducted by Student's t-test and one-way analysis of variance followed by Tukey's post hoc test, respectively. $\mathrm{P}<0.05$ was considered to indicate a statistically significant difference.

\section{Results}

DCR-3 expression levels in myocardial cells of patients with coronary heart disease. In the present study, the plasma concentration levels of DCR-3 in patients with coronary heart disease were analyzed. As presented in Fig. 1A, it was observed that plasma concentration levels of DCR-3 were downregulated in peripheral blood with the severity of the disease increasing, as determined by ELISA. In addition, it was also demonstrated that mRNA and protein expression in myocardial cells were decreased with increasing disease severity, as determined by RT-qPCR and western blotting, respectively (Fig. 1B and C). Furthermore, circulating DCR-3 levels were associated with the Syntax score and severity of coronary heart disease, which was demonstrated by the increase in SXscore along with more serious disease condition (Fig. 1D). Taken together, these results suggest DCR-3 expression levels can predict the events and severity of coronary heart disease.

DCR-3 has benefits for the treatment of mice with coronary heart disease by improvement of coronary lesions. To investigate the efficacy of DCR-3 for coronary heart disease, the therapeutic effects of DCR-3 were analyzed in mice with coronary heart disease. By analyzing the DCR-3 expression in myocardial cells, it was observed that DCR-3 expression in myocardial cells was analyzed. It was observed that DCR-3 expression levels were downregulated in myocardial cells isolated from mice with coronary heart disease (Fig. 2A). It was also observed that plasma concentration levels were downregulated in the coronary heart disease mice (Fig. 2B). In addition, DCR-3 treatment affected the lesions and inhibited the apoptosis of arterial vascular smooth muscle, as determined by histological analysis (Fig. 2C). Furthermore, results indicated that myocardial expression levels of injury-associated proteins cTnT and cTn I were upregulated in myocardium after treatment with DCR-3 (Fig. 2D). Taken together, these results suggest that DCR-3 is beneficial for the remission of coronary heart disease induced by TGF- $\beta 1$ through improvement of coronary lesions.

DCR-3 inhibits expression levels of inflammatory factors in myocardial cells. A previous study indicated that inflammatory responses are associated with the progression of coronary heart disease (28). Therefore, the inflammatory factor expression was analyzed in myocardial cells and tissues. As presented in Fig. 3A-E, following treatment with DCR-3, IL-6, CRP-1, serum albumin, ICAM-1 and VCAM-1 were all decreased in myocardial cells in mice with coronary heart disease, which indicated that DCR-3 suppressed the expression levels of these inflammatory factors. Furthermore, DCR-3 could significantly ameliorate the myocardial inflammation of lymphocytes as well (Fig. 3F). In addition, the increased expression of DCR-3 was confirmed by western blotting in myocardial cells treated 


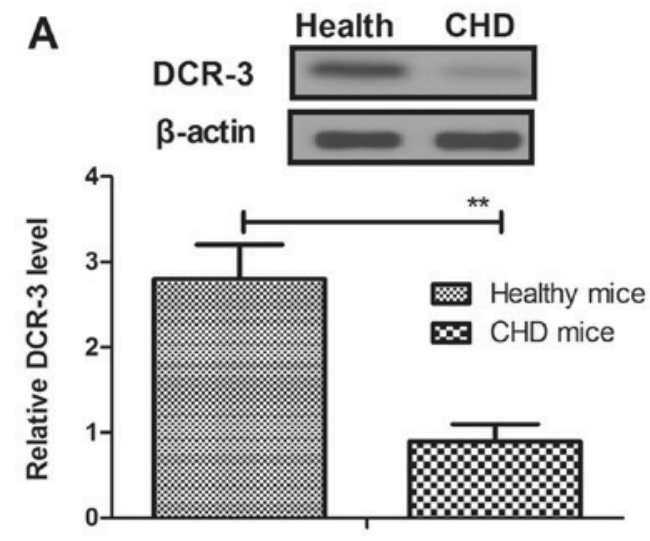

C

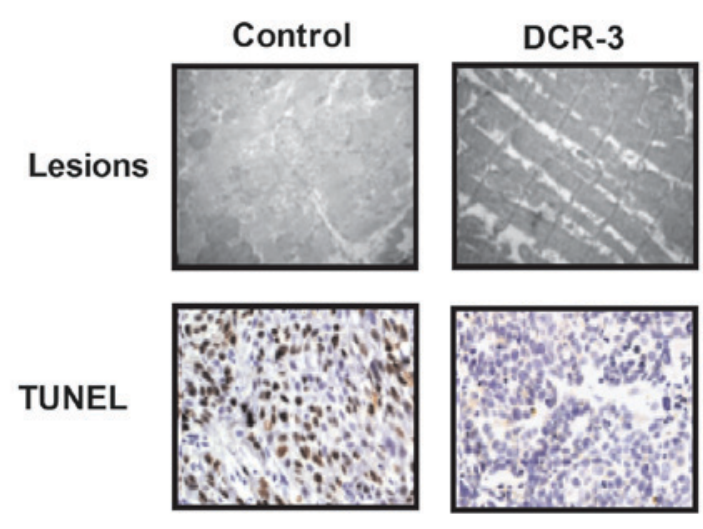

B

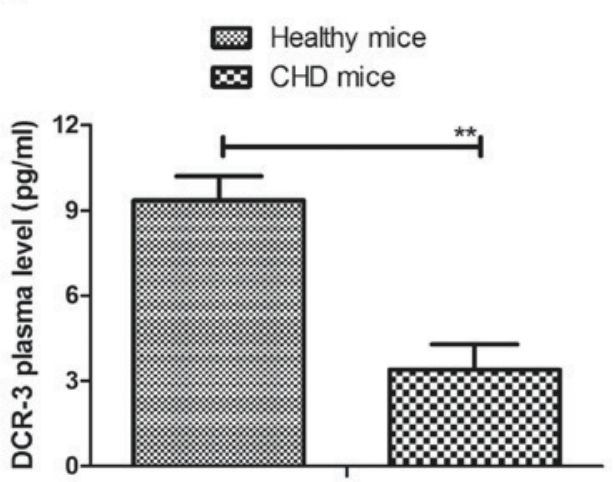

D

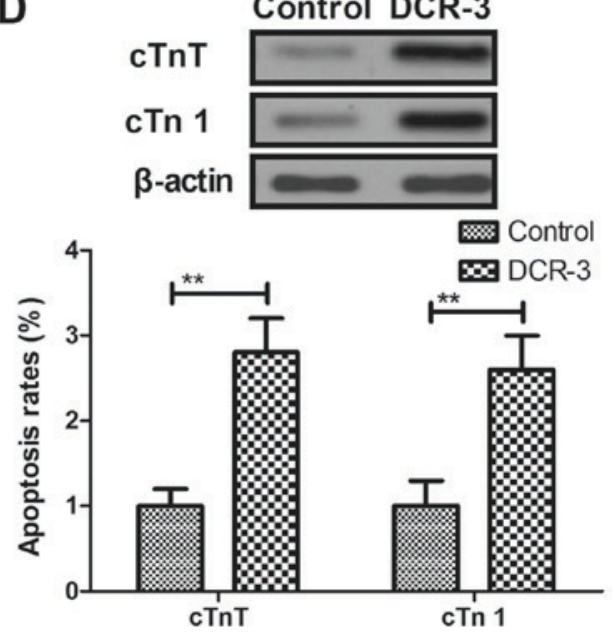

Figure 2. In vivo effects of DCR-3 on coronary lesions in mice with coronary heart disease. (A) DCR-3 expression levels in myocardial cells in mice with coronary heart disease. (B) Plasma concentration levels of DCR-3 in mice with coronary heart disease. (C) Apoptosis of arterial vascular smooth muscle and myocardial lesions in experimental mice determined by histological analysis (magnification, x400). (D) Expression levels of cTnT and cTn1 in myocardium by the treatment of DCR-3. Data are presented as the mean + standard deviation of three independent experiments. * $\mathrm{P}<0.01$. DCR-3, decoy receptor-3; cTn, cardiac troponin; CHD, coronary heart disease; TUNEL, terminal deoxynucleotidyl-transferase-mediated dUTP nick end labeling.

with DCR-3 (Fig. 3G). Collectively, these results suggest that DCR-3 can inhibit expression levels of inflammatory factors in myocardial cells in experimental mice with coronary heart disease.

DCR-3 improves inflammatory and apoptosis in myocardial cells through the PI3K/AKT signaling pathway. It has been demonstrated previously that pretreatment of inflammatory factors by activating the PI3K/AKT signaling pathway contributes to recovery of coronary heart disease-induced myocardial ischemia and injury (29). In the present study, the PI3K/AKT signaling pathway in myocardial cells was analyzed in experimental mice with coronary heart disease treated by DCR-3. As presented in Fig. 4A and B, DCR-3 in myocardial cells upregulated the expression levels of PI3K and p-AKT/AKT, compared with the control group. The results in Fig. 4C demonstrated that endogenous inhibition of PI3K expression by Si-PI3K was successfully achieved in myocardial cells. In vitro assays demonstrated that endogenous inhibition of PI3K expression by Si-PI3K suppressed AKT expression in myocardial cells (Fig. 4D). Furthermore, representative histological images revealed that Si-PI3K also markedly inhibited improvement of DCR-3-mediated apoptosis in myocardial cells, as determined by TUNEL and immunofluorescence (Fig. 4E and F). These findings suggest that PI3K/AKT signaling pathway involves the anti-inflammatory and anti-apoptosis potential of DCR-3 in myocardial cells in the progression of coronary heart disease induced by TGF- $\beta 1$.

DCR-3 prolongs survival of mice with coronary heart disease. The efficacy for the survival of mice with coronary heart disease following treatment with DCR-3 was then determined. As presented in Fig. 5, DCR-3 significantly prolonged the survival of mice with coronary heart disease induced by TGF- $\beta 1$ in a 90 -day observation ( $\mathrm{n}=20$ in each group). Survival analysis suggests that DCR-3 may be an efficient agent for the treatment of coronary heart disease.

\section{Discussion}

DCR-3 acts as a soluble receptor of Fas ligand (FasL), TNF superfamily member 14 (LIGHT) and TNF superfamily member 15 that is reported highly expressed in cancer cells, but is expressed less in myocardial cells (30). A previous study has indicated that expression of DCR-3 is notable in chronic liver disease (31). However, the significance of expression of DCR-3 
A

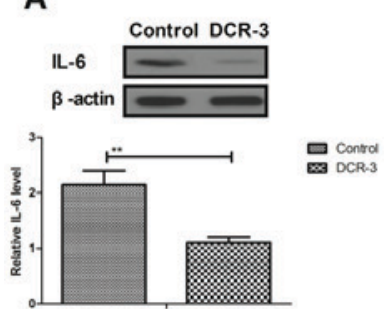

D
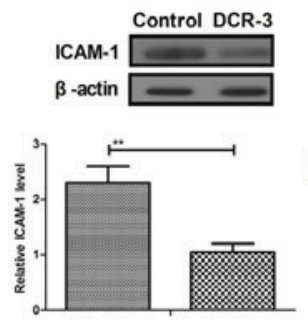

品 ${ }^{\text {Conspd }}$
B

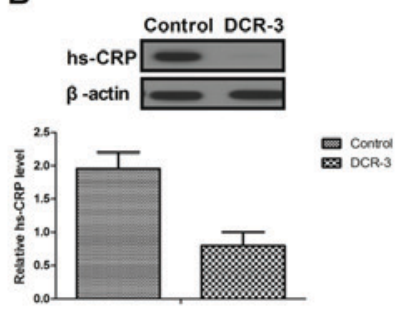

E

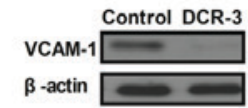

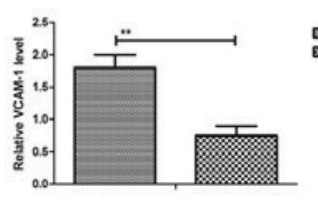

C

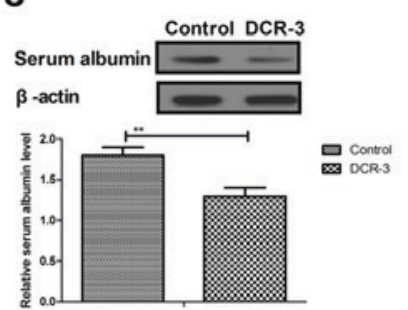

G

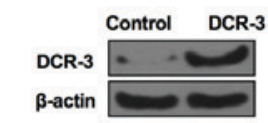

$\mathbf{F}$

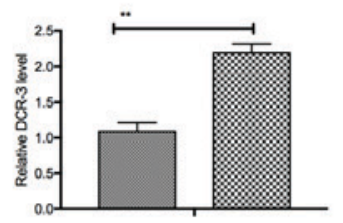

$\square$ Control DO DCR-3

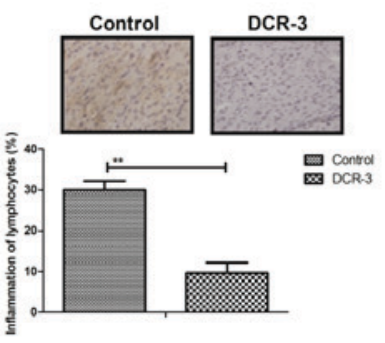

Figure 3. Expression levels of inflammatory factors in myocardial cells in mice with coronary heart disease treated by DCR-3. Protein expression levels of (A) IL-6, (B) CRP-1, (C) serum albumin, (D) ICAM-1 and (E) VCAM-1 in myocardial cells in mice with coronary heart disease treated by DCR-3. (F) Analysis of myocardial inflammation of lymphocytes in myocardial cells in mice with coronary heart disease treated by DCR-3 (magnification, x400). (G) The expression of DCR-3 was detected by western blotting in myocardial cells treated by DCR-3. Data are presented as the mean \pm standard deviation of three independent experiments. ${ }^{* *} \mathrm{P}<0.01$. DCR-3, decoy receptor-3; IL, interleukin; CRP-1, C-reactive protein 1; ICAM-1, intercellular adhesion molecule-1; VCAM-1, vascular cell adhesion molecule-1.

A

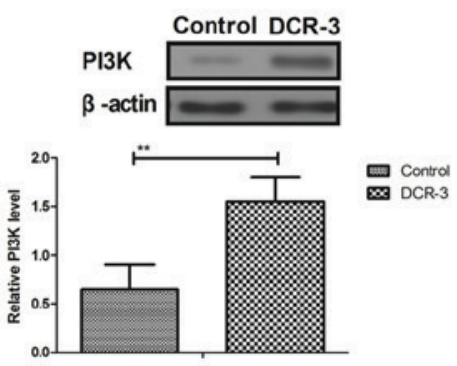

D

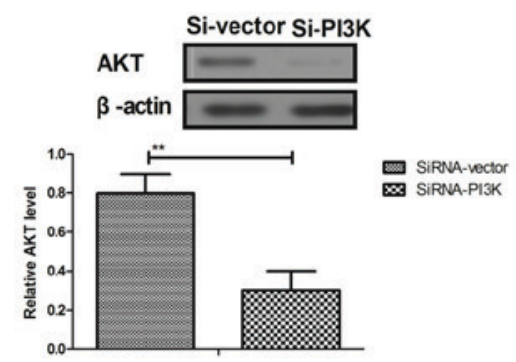

B

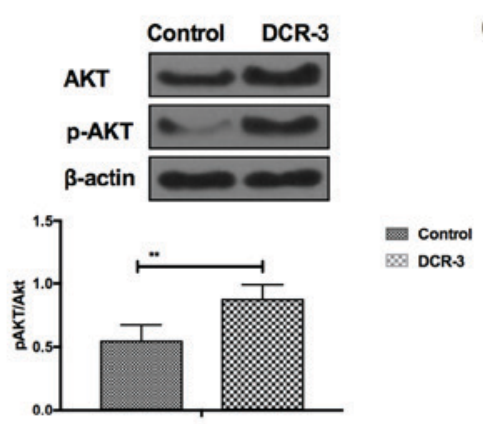

$\mathbf{E}$

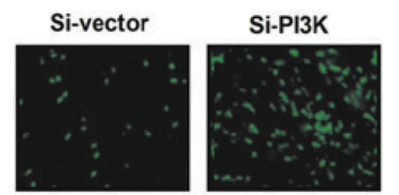

C

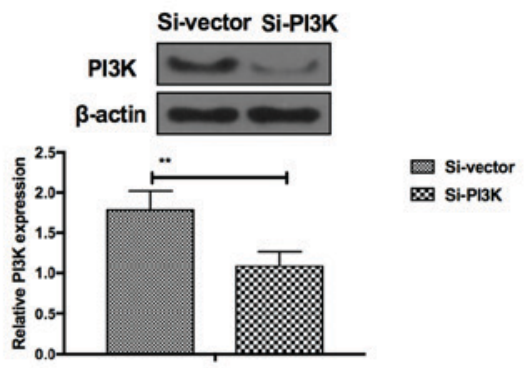

$\mathbf{F}$

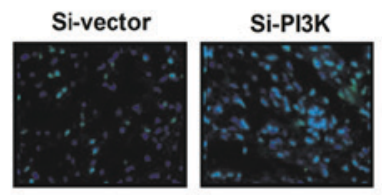

Figure 4. DCR-3 regulates inflammatory and apoptosis factors in myocardial cells through PI3K/AKT signaling pathway. Expression levels of (A) PI3K and (B) phosphorylation levels of AKT in myocardial cells in experimental mice with coronary heart disease treated by DCR-3. (C) The expression of PI3K was detected by western blotting in myocardial cells with endogenous inhibition of PI3K expression. (D) AKT expression levels in myocardial cells featured endogenous inhibition of PI3K expression. (E) Endogenous inhibition of PI3K expression disturbed DCR-3-mediated apoptosis determined by terminal deoxynucleotidyl-transferase-mediated dUTP nick end labeling (magnification, x10). (F) Endogenous inhibition of PI3K expression disturbed DCR-3-mediated apoptosis in myocardial determined by immunofluorescence. Data are presented as the mean \pm standard deviation of three independent experiments. ${ }^{* *} \mathrm{P}<0.01$. DCR-3, decoy receptor-3; PI3K, phosphoinositide 3-kinase; AKT, protein kinase B; Si, small interfering RNA.

in myocardial cells in progression of coronary heart disease has not been reported in a previous study (32). The present study investigated the molecular mechanism of DCR-3 in myocardial cells in the progression of coronary heart disease. Although previous reports have reported that plasma DCR-3 levels may indicate the severity of coronary heart disease (33), 


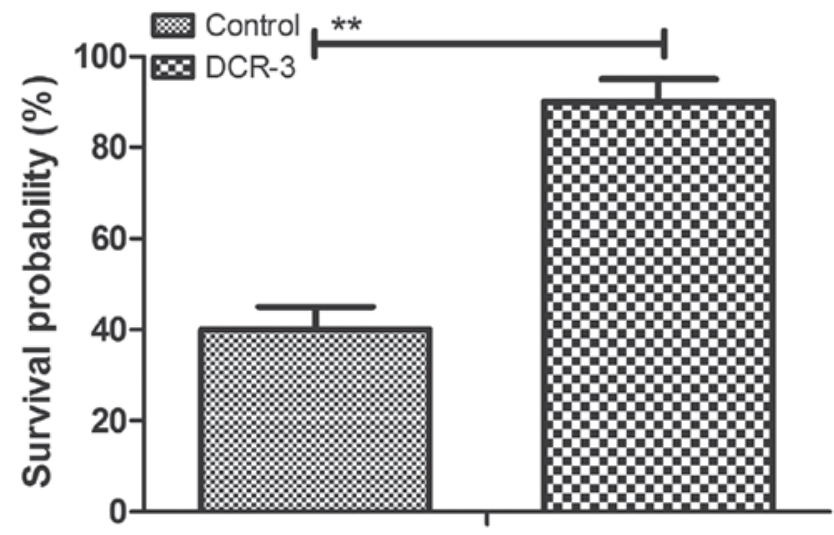

Figure 5. Survival of mice with coronary heart disease following received treatment with DCR-3. Data are presented as the mean + standard deviation of three independent experiments. ${ }^{* *} \mathrm{P}<0.01$. DCR-3, decoy receptor-3.

the potential mechanism mediated by DCR-3 has not been reported in myocardial cells. The present results demonstrated that DCR-3 is downregulated in patients with coronary heart disease and increasing DCR-3 expression can prevent inflammation and apoptosis in myocardial cells induced by coronary heart disease. DCR-3 also mediate anti-inflammatory and anti-apoptosis potential through the PI3K/AKT signaling pathway in myocardial cells in the progression of coronary heart disease induced by TGF- $\beta 1$. These findings may elucidate the function of DCR-3 in processes of apoptosis and inflammation for patients with coronary heart disease.

Inflammation is one of the most common symptoms of patients with acute coronary heart disease and the marked inflammation during acute coronary syndrome contributes to later depression in a subset of patients (34). Inflammatory response may be used as a prognostic indicator for borderline lesion coronary heart disease patients, who were treated with naoxintong (35). In addition, El-Mesallamy et al (36) have indicated that inflammation is one of factors in the imitation of coronary heart disease. Myocardial tissue has a higher level of inflammatory factors and lymphocytes. However, a stable DCR-3 analogue can reduce FasL-induced murine pulmonary inflammation (37). At the same time, DCR-3 may improve experimental autoimmune encephalomyelitis by directly counteracting local inflammation, suggesting that DCR-3 is the potential agent for treating human multiple sclerosis (38). All of these reports suggest that DCR-3 is associated with inflammatory responses in patients with cardiovascular disease. Additionally, although previous data demonstrated that slightly elevated cTnS levels may not be a sensitive prognostic marker for the Chinese population (39), a number of studies have indicated that serum cTnT levels are also regarded as an indicator of myocardial injury in ischemic and hemorrhagic stroke patients (40-42). The present results demonstrated that DCR-3 increased serum levels of cTnT and cTn1 in mice with coronary heart disease and that DCR-3 can inhibit inflammation responses and expression levels of inflammatory responses in myocardial cells in mice with coronary heart disease.

Apoptosis of myocardial cells is another crucial factor in initiating coronary heart disease (7). Considerable evidence has indicated that apoptosis has an important role in hepatocyte death in chronic liver disease (43). Research also demonstrated that apoptosis of leukocytes acts as a disease marker of neutrophil-endotheliocyte interaction in patients with coronary heart disease (14). Liu et al (15) have previously investigated the expression levels of inflammatory and apoptosis factors in coronary stent implantation in coronary heart disease patients, and demonstrated that downregulation of inflammatory and apoptosis factors is beneficial to the recovery of coronary heart disease. DCR-3, as a pleiotropic immunomodulator for enhancing angiogenesis, has been observed to downregulate cell apoptosis by binding with its receptor of FasL and LIGHT, and subsequently improved survival of many types of cell, such as human IPF fibroblasts (44) and mouse lymphocytes (45). Furthermore, DCR-3 also suppressed FasL-induced apoptosis via extracellular signal-regulated kinase $1 / 2$ activation in various cancer cells (46-48). Furthermore, a number of studies have reported DCR-3 function in lymphocytes and dendritic cells $(45,49)$. The present study indicated that DCR-3 could inhibit apoptosis of myocardial cells in mice with coronary heart disease, which contributed to protect myocardial cells against injury in the process of coronary heart disease. Notably, injury-associated protein levels of cTnT and cTn I were markedly upregulated in myocardium by the treatment of DCR-3. These results suggest that inhibition of apoptosis is the essential function of DCR-3 for the treatment of coronary heart disease. However, the present study did not use a healthy or sham control to identify the role of DCR-3 for the treatment of coronary heart disease, which is a limitation of this research.

The PI3K/AKT signaling pathway is involved in the progression of myocardial infarction in the process of coronary heart disease (50). Hu et al (51) presented the protective effect of proanthocyanidins on anoxia-reoxygenation injury of myocardial cells and results have indicated that the PI3K/AKT/glycogen synthase kinase-3 $\beta$ pathway mediates mitochondrial ATP-sensitive potassium channel to regulate oxidative stress. It has also been indicated that the PI3K/AKT pathway-regulated recruiting $\mathrm{T}$ cells could attenuate myocardial ischemia-reperfusion injury in a mice model (52). In the present study, by investigating the association between DCR-3 and the PI3K/AKT signal pathway in myocardial cells in mice with coronary heart disease, it was observed that DCR-3 mediated the improvement of myocardial ischemia via the PI3K/AKT signaling pathway. Notably, it also demonstrated that endogenous inhibition of PI3K expression by Si-PI3K abolished DCR-3-mediated anti-inflammation and anti-apoptosis in myocardial cells in mice with coronary heart disease.

In conclusion, the present study investigated the efficacy of DCR-3 in the progression of coronary heart disease and the molecular mechanism of DCR-3-mediated anti-inflammation and anti-apoptosis in myocardial cells in mice with coronary heart disease. Results have demonstrated that patients and mice with coronary heart disease have lower DCR-3 expression levels in peripheral blood. DCR-3 can inhibit inflammatory factor expression and apoptosis of myocardial cells and that the PI3K/AKT signaling pathway is associated with anti-inflammation and anti-apoptosis in the process of coronary heart disease. These results confirm that DCR-3 treatment may be beneficial for the recovery of coronary heart disease by anti-inflammation and anti-apoptosis in myocardial cells through the PI3K/AKT signaling pathway, suggesting that DCR-3 may be able to predict the extent and severity of cardiovascular diseases. 


\section{Acknowledgements}

Not applicable.

\section{Funding}

The present study was supported by grants from Fujian Provincial Health and Family Planning Young and Middle-aged Key Members Talent Training Project (grant no. 2017-ZQN-9), Fujian Provincial Natural Science Funding Project (grant no. 2018J01244), The Key Clinical Specialty Discipline Construction Program of Fujian (Program of Vasculocardiology) and Fujian Provincial Youth Scientific Research Project of Health Department (grant no. 2012-2-10).

\section{Availability of data and materials}

The datasets used and/or analyzed during the current study are available from the corresponding author on reasonable request.

\section{Authors' contributions}

XJC, RHW and WC designed the experiments. XJC and RHW performed the experiments and analysis. RHW and LL helped analyze the data. RHW, WC and ZLL wrote the manuscript. All authors read and approved the final manuscript.

\section{Ethics approval and consent to participate}

The present study was approved by the Ethics Committee and the Committee for Experimental Animal Studies of Southern Medical University (Guangzhou, China). All patients provided written informed consent.

\section{Patient consent for publication}

All patients provided written informed consent.

\section{Competing interests}

The authors declare that they have no competing interests.

\section{References}

1. Tully PJ and Baumeister H: Collaborative care for comorbid depression and coronary heart disease: A systematic review and meta-analysis of randomised controlled trials. BMJ Open 5: e009128, 2015.

2. Heikkilä K, Koskinen OA, Agarwal A, Tikkinen KA, Mäki M and Kaukinen K: Associations of coeliac disease with coronary heart disease and cerebrovascular disease: A systematic review and meta-analysis. Nutr Metab Cardiovasc Dis 25: 816-831, 2015.

3. Mayor S: Reduced blood pressure and cholesterol are main factors in fall in deaths from coronary heart disease. BMJ 350: h415, 2015.

4. Niermann C, Gorressen S, Klier M, Gowert NS, Billuart P, Kelm M, Merx MW and Elvers M: Oligophrenin1 protects mice against myocardial ischemia and reperfusion injury by modulating inflammation and myocardial apoptosis. Cell Signal 28: 967-978, 2016.

5. Guo CX, Jiang X, Zeng XJ, Wang HX, Li HH, Du FH and Chen BX: Soluble receptor for advanced glycation end-products protects against ischemia/reperfusion-induced myocardial apoptosis via regulating the ubiquitin proteasome system. Free Radic Biol Med 94: 17-26, 2016.
6. O'Neal WT, Soliman EZ, Howard G, Howard VJ, Safford MM, Cushman $M$ and Zakai NA: Inflammation and hemostasis in atrial fibrillation and coronary heart disease: The REasons for geographic and racial differences in stroke study. Atherosclerosis 243: 192-197, 2015.

7. Geng YJ: Molecular mechanisms for cardiovascular stem cell apoptosis and growth in the hearts with atherosclerotic coronary disease and ischemic heart failure. Ann N Y Acad Sci 1010: 687-697, 2003.

8. Fang Y, Chen S, Liu Z, Ai W, He X, Wang L, Xie P, Jiang B and Fang H: Endothelial stem cells attenuate cardiac apoptosis via downregulating cardiac microRNA-146a in a rat model of coronary heart disease. Exp Ther Med 16: 4246-4252, 2018.

9. Xu L, Jiang X, Wei F and Zhu H: Leonurine protects cardiac function following acute myocardial infarction through anti-apoptosis by the PI3K/AKT/GSK3 $\beta$ signaling pathway. Mol Med Rep 18: 1582-1590, 2018.

10. Wang Z, Zhang J, Ren T and Dong Z: Targeted metabolomic profiling of cardioprotective effect of Ginkgo biloba L. extract on myocardial ischemia in rats. Phytomedicine: international journal of phytotherapy and phytopharmacology 23: 621-631, 2016.

11. Wang L, Niu X, Hu J, Xing H, Sun M, Wang J, Jian Q and Yang H: After myocardial ischemia-reperfusion, mir-29a, and let7 could affect apoptosis through regulating IGF-1. BioMed Research International 2015: 245412, 2015.

12. Wakiyama H, Cowan DB, Toyoda Y, Federman M, Levitsky S and McCully JD: Selective opening of mitochondrial ATP-sensitive potassium channels during surgically induced myocardial ischemia decreases necrosis and apoptosis. Eur J Cardiothorac Surg 21: 424-433, 2002

13. Elsasser A, Suzuki K, Lorenz-Meyer S, Bode C and Schaper J: The role of apoptosis in myocardial ischemia: A critical appraisal. Basic Res Cardiol 96: 219-226, 2001.

14. Salmina AB, Shul'man VA, Nikulina SY, Trufanova LV, Fursov AA, But'yanov PA, Kuskaev AP, Bol'shakova EV and Kotlovskii MY: Apoptosis of leukocytes as a marker of neutrophil-endotheliocyte interaction in coronary heart disease. Bull Exp Biol Med 144: 39-41, 2007 (In English, Russian).

15. Liu LL, Lin LR, Lu CX, Fu JG, Chao PL, Jin HW, Zhang ZY and Yang TC: Expression of inflammatory and apoptosis factors following coronary stent implantation in coronary heart disease patients. Int Immunopharmacol 11: 1850-1854, 2011.

16. Azambuja MI: Inflammation as the cause of coronary heart disease. Lancet Infect Dis 10: 142-143, 2010.

17. Wu T, Sun QF, Yang PS, Feng W and Liu XL: Levels of inflammation cytokines in patients with coronary heart disease and periodontal disease. Zhonghua Kou Qiang Yi Xue Za Zhi 45: 265-268, 2010 (In Chinese).

18. Yan Y, Song D, Liu L, Meng X, Qi C and Wang J: The relationship of plasma decoy receptor 3 and coronary collateral circulation in patients with coronary artery disease. Life Sci 189: 84-88, 2017.

19. Marriott HM, Daigneault M, Thompson AA, Walmsley SR, Gill SK, Witcher DR, Wroblewski VJ, Hellewell PG, Whyte MK and Dockrell DH: A decoy receptor 3 analogue reduces localised defects in phagocyte function in pneumococcal pneumonia. Thorax 67: 985-992, 2012.

20. Wu SF, Liu TM, Lin YC, Sytwu HK, Juan HF, Chen ST, Shen KL, his SC and Hsieh SL: Immunomodulatory effect of decoy receptor 3 on the differentiation and function of bone marrow-derived dendritic cells in nonobese diabetic mice: From regulatory mechanism to clinical implication. J Leukoc Biol 75: 293-306, 2004

21. Jiang M, Lin X, He R, Lin X, Liang L, Tang R, Xiong D, Wei K, Dang Y, Feng Z and Chen G: Decoy receptor 3 (DcR3) as a biomarker of tumor deterioration in female reproductive cancers: A meta-analysis. Med Sci Monit 22: 1850-1857, 2016.

22. Dietz R and Rauch B; German Society of Cardiology-Heart Circulation Research; German Society for Prevention and Rehabilitation of Cardiac Diseases; German Society for Thoracic and CardiovascularSurgery: Guidelines for diagnosis and treatment of chronic coronary heart disease. Issued by the executive committee of the German Society of Cardiology - Heart Circulation Research in cooperation with the German Society for Prevention and Rehabilitation of Cardiac Diseases and the German Society for Thoracic and Cardiovascular Surgery. Z Kardiol 92: 501-521, 2003 (In German).

23. Golden HB, Gollapudi D, Gerilechaogetu F, Li J, Cristales RJ, Peng X and Dostal DE: Isolation of cardiac myocytes and fibroblasts from neonatal rat pups. Methods Mol Biol 843: 205-214, 2012. 
24. Lee I, Chalon J, Ramanathan S, Gross S and Turndorf H: Analgesic properties of meperidine, amitriptyline and phenelzine in mice. Can Anaesth Soc J 30: 501-505, 1983.

25. Livak KJ and Schmittgen TD: Analysis of relative gene expression data using real-time quantitative PCR and the 2(-Delta Delta C(T)) method. Methods 25: 402-408, 2001.

26. Börekçi A, Gür M, Şeker T, Baykan AO, Özaltun B, Karakoyun S, Karakurt A, Türkoğlu C, Makça I and Çaylı M: Coronary collateral circulation in patients with chronic coronary total occlusion; its relationship with cardiac risk markers and SYNTAX score. Perfusion 30: 457-464, 2015

27. Wu ZW, Liu YF, Wang S and Li B: miRNA-146a induces vascular smooth muscle cell apoptosis in a rat model of coronary heart disease via NF-kB pathway. Genet Mol Res 14: 18703-18712, 2015.

28. Werba JP, Veglia F, Amato M, Baldassarre D, Massironi P, Meroni PL, Riboldi P, Tremoli E and Camera M: Patients with a history of stable or unstable coronary heart disease have different acute phase responses to an inflammatory stimulus. Atherosclerosis 196: 835-840, 2008.

29. Rong R and Xijun X: Erythropoietin pretreatment suppresses inflammation by activating the PI3K/Akt signaling pathway in myocardial ischemia-reperfusion injury. Exp Ther Med 10: 413-418, 2015

30. Funke B, Autschbach F, Kim S, Lasitschka F, Strauch U, Rogler G, Gdynia G, Li L, Gretz N, Macher-Goeppinger S, et al: Functional characterisation of decoy receptor 3 in Crohn's disease. Gut 58 : 483-491, 2009.

31. Kim S, Kotoula V, Hytiroglou P, Zardavas D and Zhang L: Significance of increased expression of decoy receptor 3 in chronic liver disease. Dig Liver Dis 41: 591-598, 2009.

32. Chang TY, Hsu CY, Huang PH, Chiang CH, Leu HB, Huang CC, Chen JW and Lin SJ: Usefulness of circulating decoy receptor 3 in predicting coronary artery disease severity and future major adverse cardiovascular events in patients with multivessel coronary artery disease. Am J Cardiol 116: 1028-1033, 2015.

33. Kim S, McAuliffe WJ, Zaritskaya LS, Moore PA, Zhang L and Nardelli B: Selective induction of tumor necrosis receptor factor $6 /$ decoy receptor 3 release by bacterial antigens in human monocytes and myeloid dendritic cells. Infect Immun 72: 89-93, 2004

34. Steptoe A, Wikman A, Molloy GJ, Messerli-Bürgy N and Kaski JC: Inflammation and symptoms of depression and anxiety in patients with acute coronary heart disease. Brain Behav Immun 31: 183-188, 2013.

35. Li SR, Wang TH and Zhang BJ: Effects of naoxintong capsule on the inflammation and prognosis in borderline lesion coronary heart disease patients. Zhongguo Zhong Xi Yi Jie He Za Zhi 32: 607-611, 2012 (In Chinese).

36. El-Mesallamy HO, Hamdy NM, Salman TM and Ibrahim SM Adiponectin and sE-selectin concentrations in relation to inflammation in obese type 2 diabetic patients with coronary heart disease. Angiology 63: 96-102, 2012.

37. Wortinger MA, Foley JW, Larocque P, Witcher DR, Lahn M, Jakubowski JA, Glasebrook A and Song HY: Fas ligand-induced murine pulmonary inflammation is reduced by a stable decoy receptor 3 analogue. Immunology 110: 225-233, 2003.

38. Chen SJ, Wang YL, Kao JH, Wu SF, Lo WT, Wu CC, Tao PL, Wang CC, Chang DM and Sytwu HK: Decoy receptor 3 ameliorates experimental autoimmune encephalomyelitis by directly counteracting local inflammation and downregulating Th17 cells. Mol Immunol 47: 567-574, 2009.

39. Zhang M, He H, Wang ZM, Xu Z, Zhou N, Tao Z, Chen B, Li C, Zhu T, Yang D, et al: Diagnostic and prognostic value of minor elevated cardiac troponin levels for percutaneous coronary intervention-related myocardial injury: A prospective, single-center and double-blind study. J Biomed Res 28: 98-107, 2014.
40. Apak I, Iltumur K, Tamam Y and Kaya N: Serum cardiac troponin $\mathrm{T}$ levels as an indicator of myocardial injury in ischemic and hemorrhagic stroke patients. Tohoku J Exp Med 205: 93-101, 2005.

41. Nesher N, Zisman E, Wolf T, Sharony R, Bolotin G, David M, Uretzky G and Pizov R: Strict thermoregulation attenuates myocardial injury during coronary artery bypass graft surgery as reflected by reduced levels of cardiac-specific troponin I. Anesth Analg 96: 328-335, 2003

42. Boriani G, Biffi M, Cervi V, Bronzetti G, Magagnoli G, Zannoli R and Branzi A: Evaluation of myocardial injury following repeated internal atrial shocks by monitoring serum cardiac troponin I levels. Chest 118: 342-347, 2000

43. Wu ZW, Liu YF, Wang S and Li B: Corrigendum miRNA-146a induces vascular smooth muscle cell apoptosis in a rat model of coronary heart disease via NF-kappaB pathway - Genet. Mol. Res. 14 (4): 18703-18712. Genet Mol Res 15, 2016.

44. Im J, Kim K, Hergert P and Nho RS: Idiopathic pulmonary fibrosis fibroblasts become resistant to Fas ligand-dependent apoptosis via the alteration of decoy receptor 3. J Pathol 240: 25-37, 2016

45. Liang D, Hou Y, Lou X and Chen H: Decoy receptor 3 improves survival in experimental sepsis by suppressing the inflammatory response and lymphocyte apoptosis. PLoS One 10: e0131680, 2015.

46. Zhang Y, Li D, Zhao X, Song S, Zhang L, Zhu D, Wang Z, Chen $X$ and Zhou J: Decoy receptor 3 suppresses FasL-induced apoptosis via ERK1/2 activation in pancreatic cancer cells. Biochem Biophys Res Commun 463: 1144-1151, 2015.

47. Koksal IT, Sanlioglu AD, Karacay B, Griffith TS and Sanlioglu S: Tumor necrosis factor-related apoptosis inducing ligand-R4 decoy receptor expression is correlated with high Gleason scores, prostate-specific antigen recurrence, and decreased survival in patients with prostate carcinoma. Urol Oncol 26: 158-165, 2008.

48. Zhou J, Song S, He S, Wang Z, Zhang B, Li D and Zhu D Silencing of decoy receptor 3 (DcR3) expression by siRNA in pancreatic carcinoma cells induces Fas ligand-mediated apoptosis in vitro and in vivo. Int J Mol Med 32: 653-660, 2013.

49. You RI, Chang YC, Chen PM, Wang WS, Hsu TL, Yang CY, Lee CT and Hsieh SL: Apoptosis of dendritic cells induced by decoy receptor 3 (DcR3). Blood 111: 1480-1488, 2008.

50. Zhang Z, Li S, Cui M, Gao X, Sun D, Qin X, Narsinh K, Li C, Jia H, Li C, et al: Rosuvastatin enhances the therapeutic efficacy of adipose-derived mesenchymal stem cells for myocardial infarction via PI3K/Akt and MEK/ERK pathways. Basic Res Cardiol 108: 333, 2013.

51. Hu Y, Li L, Yin W, Shen L, You B and Gao H: Protective effect of proanthocyanidins on anoxia-reoxygenation injury of myocardial cells mediated by the PI3K/Akt/GSK-3beta pathway and mitochondrial ATP-sensitive potassium channel. Mol Med Rep 10: 2051-2058, 2014.

52. Fang J, Hu F, Ke D, Yan Y, Liao Z, Yuan X, Wu L, Jiang Q and Chen L: N,N-dimethylsphingosine attenuates myocardial ischemia-reperfusion injury by recruiting regulatory T cells through PI3K/Akt pathway in mice. Basic Res Cardiol 111: 32, 2016.

This work is licensed under a Creative Commons Attribution-NonCommercial-NoDerivatives 4.0 International (CC BY-NC-ND 4.0) License. 